\title{
Wavelet Denoising of Vehicle Platform Vibration Signal Based on Threshold Neural Network
}

\author{
Mingzhu Li, ${ }^{1,2}$ Zhiqian Wang, ${ }^{1}$ Jun Luo, ${ }^{1,2}$ Yusheng Liu, ${ }^{1,2}$ and Sheng Cai ${ }^{1}$ \\ ${ }^{1}$ Changchun Institute of Optics, Fine Mechanics and Physics, Chinese Academy of Sciences, Changchun, Jilin, China \\ ${ }^{2}$ University of Chinese Academy of Sciences, Beijing, China
}

Correspondence should be addressed to Zhiqian Wang; wangzhiqian2000@gmail.com

Received 27 June 2016; Revised 5 December 2016; Accepted 20 December 2016; Published 26 January 2017

Academic Editor: Mickaël Lallart

Copyright (C) 2017 Mingzhu Li et al. This is an open access article distributed under the Creative Commons Attribution License, which permits unrestricted use, distribution, and reproduction in any medium, provided the original work is properly cited.

Vehicle Platform Vibration Signal (VPVS) denoising is essential to achieve high measurement accuracy of precise optical measuring instrument (POMI). A method to denoise the VPVS is proposed based on the wavelet coefficients thresholding and threshold neural network (TNN). According to the characteristics of VPVS, a novel thresholding function is constructed, and then its optimized threshold is selected through unsupervised learning of TNN. The original VPVS mixed in trend and random noise is constructed as VPVS model. A VPVS denoising flow is proposed based on the power spectral and energy distribution of the VPVS model. The simulation shows that the proposed denoising method achieves better results, compared to the previous denoising methods using the indexes of SNR and RMSE. The experiment demonstrates that it is efficient for denoising VPVS polluted by the trend and random noise.

\section{Introduction}

VPVS denoising is essential to guarantee the measurement accuracy of POMI. In project, POMI is installed on the vehicle platform to ensure its mobility. However, its measuring basis is easily influenced by the engine and generator vibration, and the solution is to compensate its measured data with VPVS. During the VPVS collection, the actual VPVS is polluted by complex noise, especially trend and random noise. Thus, it is necessary to remove the trend and random noise from the polluted VPVS.

Wavelet threshold denoising method is efficient for VPVS denoising on account of its good localization property in the domain of time and frequency. The basic wavelet threshold denoising method is Donoho's hard and soft thresholding method [1], which is the most common VPVS denoising method. The estimated wavelet coefficients processed by hard thresholding function have a bad continuity, causing the oscillation and poor smoothness of the reconstructed signal. Meanwhile, there are deviations between the estimated wavelet coefficients processed by soft thresholding function and the actual wavelet coefficients, which brings out the distortion of the reconstructed signal [2]. So far, the improved algorithms of soft and hard thresholding method are rarely aimed at VPVS denoising, but it is valuable to refer to the improved wavelet threshold denoising algorithms in other fields.

So far, the improved algorithms mainly focus on two aspects: the establishment of the thresholding function and the selection of the wavelet threshold. For the latter, some machine learning methods can be referred to obtain the wavelet threshold. Gu et al. proposed some improved classification methods to get the better effectiveness, such as structural minimax probability machine, incremental support vector ordinal regression, and $v$-support vector classification [3-5]. Xia and Wen applied the improved classification methods to the field of image $[6,7]$. For the cluster methods, Deng et al. proposed an improved ant colony optimization algorithm to get strong flexibility, adaptability, and robustness $[8,9]$. Zheng et al. improved the Fuzzy $C$-means algorithm to achieve better robustness and effectiveness [10]. These methods can be improved to obtain the optimal threshold.

Considering the characteristics of the VPVS signal, TNN is a more suitable method to select the wavelet threshold. TNN is different from the traditional neural networks; the input and output weights are fixed to one; in other words, 
the wavelet threshold denoising of each decomposition level is independent. Zhang's TNN is an improved adaptive wavelet threshold denoising algorithm, which is used to seek the optimal wavelet threshold [11]. Nasri and Nezamabadi-pour proposed a new thresholding function adaptive to TNN, which performs better than Zhang's TNN in image denoising field [12]. Wang et al. proposed a new thresholding function, which avoids the shortcomings of the soft and hard thresholding function [13]. All of these wavelet threshold denoising methods can be applied to VPVS denoising.

In this paper, a VPVS denoising method with specific denoising flow is proposed based on TNN. The study of the VPVS denoising method includes two parts: establishing a thresholding function as the activation function of TNN and selecting the threshold through unsupervised learning of TNN. According to the characteristics of VPVS, two VPVS models are constructed to ensure the simulation rigor. As for VPVS denoising flow, VPVS is filtered into the highfrequency part and the low-frequency part based on the power spectral distribution and energy distribution of the VPVS models; each part is decomposed and reconstructed, respectively; the detail coefficients of both parts are processed by TNN; the approximate coefficients of high-frequency part are reserved and the low-frequency ones are set to zero; the denoised signal is obtained through the superposition of the two denoised parts. Both simulative and actual experiments demonstrate that it is efficient for denoising VPVS polluted by the trend and random noise.

This paper is organized as follows. Section 2 presents the theoretical background of wavelet threshold denoising method and the evaluation criterion of noise reduction. In Section 3, the new thresholding function and the threshold selection through unsupervised learning of TNN are presented. The establishment of VPVS models and their power spectral and energy distribution are explained in Section 4. The VPVS denoising flow and simulation are discussed in Section 5. Section 6 presents the experimental results. The conclusion is presented in Section 7.

\section{Wavelet Threshold Denoising Method and Evaluation Criterion}

2.1. Wavelet Threshold Denoising Method. The theoretical basis of wavelet threshold denoising method is based on Mallat's theory; the theory considers that a signal can be completely reconstructed by its low-frequency approximation part and high-frequency detail part [14]. According to Mallat's theory, suppose an original discrete signal $s(n)$ given by

$$
s(n)=\sum_{k \in z} c_{j, k} \varphi_{j, k}(n)+\sum_{i=1}^{j} \sum_{k \in z} d_{i, k} \Psi_{i, k}(n),
$$

where $z$ is an integer, $c_{j, k}$ is the approximate coefficient, $j$ is the decomposition level, $\varphi_{j, k}(n)$ is the scaling function, $d_{i, k}$ is the detail coefficient, and $\Psi_{i, k}(n)$ is the wavelet basis function.

$c_{j, k}$ contains the low-frequency information of the original discrete signal $s(n)$, which is defined as follows:

$$
c_{j, k}=\left\langle s(n), \varphi_{j, k}(n)\right\rangle .
$$

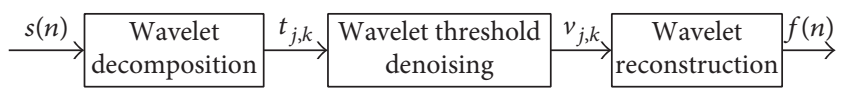

FIGURE 1: Wavelet threshold denoising process, where $s(n)$ is the original noisy signal, $t_{j, k}$ is the wavelet coefficient obtained from the wavelet decomposition of $s(n), t_{j, k}$ includes the approximate coefficient $c_{j, k}$ and the detail coefficient $d_{i, k}, v_{j, k}$ is the estimated wavelet coefficient after wavelet threshold denoising, and $f(n)$ is the estimated $s(n)$ obtained from the wavelet reconstruction of $v_{j, k}$.

$\left\langle s(n), \varphi_{j, k}(n)\right\rangle$ means the orthogonal relationship between $s(n)$ and $\varphi_{j, k}(n)$.

$d_{i, k}$ contains the high-frequency information of the original discrete signal $s(n)$, which is defined as follows:

$$
d_{i, k}=\left\langle s(n), \Psi_{i, k}(n)\right\rangle
$$

$\left\langle s(n), \Psi_{i, k}(n)\right\rangle$ means the orthogonal relationship between $s(n)$ and $\Psi_{i, k}(n)$.

Wavelet threshold denoising method considers that the low-frequency part of the signal represents its major profile, and the high-frequency part represents its details. Furthermore, its details of each level contain the noise information after wavelet decomposition. The detail coefficients $d_{i, k}$ of each level are tuned by thresholding function and reconstructed with the approximate coefficients $c_{j, k}$ of the last level. The wavelet threshold denoising process is shown in Figure 1.

2.2. Key Factors of Wavelet Threshold Denoising Method. The wavelet decomposition and reconstruction process includes two factors: the selection of wavelet basis function $\Psi_{i, k}(n)$ and decomposition level $j$. The wavelet threshold denoising process includes two factors: the selection of the threshold and thresholding function. These four factors are analyzed as follows.

(1) Wavelet Basis Function. There are some properties to be considered when selecting wavelet basis function, such as support length, symmetry, vanishing moment, and regularity [15]. Considering these properties, the suitable wavelet basis function is Daubechies $(\mathrm{db} N)$, which is an orthogonal wavelet basis function with compact support widely used in discrete wavelet transform. Its vanishing moment is $N$, and the increasing $N$ leads to the increasing regularity. db14 is used as the wavelet basis function in this paper.

(2) Maximum Decomposition Level. The method to determine the maximum decomposition level is referred to in publication [16]. It proposed that the maximum decomposition level can be determined according to the minimum frequency of the desired signal. In the experiment, the main vibration frequency range of the vehicle platform is $6 \mathrm{~Hz} \sim 65 \mathrm{~Hz}$; the maximum decomposition level is selected as 3 according to publication [16].

(3) Threshold Selection Rule. The multiresolution threshold method is used as the threshold selection rule, it considers that the wavelet coefficients of signal increase with the increasing decomposition level, while the wavelet coefficients 
of noise are opposite during the wavelet decomposition process [17].

(4) Thresholding Function. The basis thresholding functions are soft and hard thresholding functions [18]; the soft thresholding function is as follows:

$$
\eta_{s}\left(t_{j, k}, \lambda_{j}\right)= \begin{cases}t_{j, k}-\lambda_{j} \operatorname{sgn}\left(t_{j, k}\right), & \left|t_{j, k}\right| \geq \lambda_{j} \\ 0, & \left|t_{j, k}\right|<\lambda_{j},\end{cases}
$$

where $\lambda_{j}$ is threshold and sgn is sign function. The hard thresholding function is as follows:

$$
\eta_{h}\left(t_{j, k}, \lambda_{j}\right)= \begin{cases}t_{j, k}, & \left|t_{j, k}\right| \geq \lambda_{j} \\ 0, & \left|t_{j, k}\right|<\lambda_{j} .\end{cases}
$$

Zhang proposed a new thresholding function to be the activation function of TNN [11]; the improved thresholding function is as follows:

$$
\begin{aligned}
& \eta_{z}\left(t_{j, k}, \lambda_{j}\right) \\
& \quad=t_{j, k} \\
& \quad+\frac{1}{2}\left(\sqrt{\left(t_{j, k}-\lambda_{j}\right)^{2}+m}-\sqrt{\left(t_{j, k}+\lambda_{j}\right)^{2}+m}\right),
\end{aligned}
$$

where $m$ is selected as 0.5 .

Nasri and Nezamabadi-pour proposed a new thresholding function to adapt to the TNN [12]; the improved thresholding function is as follows:

$$
\eta_{n}\left(t_{j, k}, \lambda_{j}\right)= \begin{cases}t_{j, k}-\frac{0.5 \lambda_{j}^{2}}{t_{j, k}}, & \left|t_{j, k}\right| \geq \lambda_{j} \\ \frac{0.5 t_{j, k}{ }^{3}}{\lambda_{j}{ }^{2}}, & \left|t_{j, k}\right|<\lambda_{j} .\end{cases}
$$

Wang et al. proposed a new thresholding function to avoid the shortcomings of the soft and hard thresholding functions [13]; the improved thresholding function is as follows:

$$
\begin{aligned}
& \eta_{w}\left(t_{j, k}, \lambda_{j}\right) \\
& = \begin{cases}t_{j, k}-\lambda_{j} \sin \left(\frac{\pi}{2}\left|\frac{\lambda_{j}}{t_{j, k}}\right|^{n}\right), & t_{j, k} \geq \lambda_{j} \\
0, & \left|t_{j, k}\right|<\lambda_{j} \\
t_{j, k}+\lambda_{j} \sin \left(\frac{\pi}{2}\left|\frac{\lambda_{j}}{t_{j, k}}\right|^{n}\right), & t_{j, k} \leq-\lambda_{j},\end{cases}
\end{aligned}
$$

where $n$ is selected as 0.02 .

2.3. Evaluation Criterion. The wavelet denoising evaluation criterion includes two indexes: Signal Noise Ratio (SNR) and
Root Mean Squared Error (RMSE) [19]. Their equations are as follows:

$$
\begin{aligned}
\mathrm{SNR} & =10 \log _{10}\left(\frac{p_{s}}{p_{n}}\right), \\
\mathrm{RMSE} & =\sqrt{\frac{1}{N} \sum_{n=1}^{N}[s(n)-f(n)]^{2},}
\end{aligned}
$$

where $N$ is the signal data length, $p_{s}$ is desired signal power, and $p_{n}$ is noise power, The equations of $p_{s}$ and $p_{n}$ are as follows:

$$
\begin{aligned}
& p_{s}=\frac{1}{N} \sum_{n=1}^{N} s(n)^{2}, \\
& p_{n}=\frac{1}{N} \sum_{n=1}^{N}[s(n)-f(n)]^{2} .
\end{aligned}
$$

According to the definitions of SNR and RMSE, the larger the SNR, the smaller the RMSE and the better the noise reduction effect.

\section{Proposed Thresholding Function and Threshold Selection}

3.1. The Establishment of Thresholding Function. The improved thresholding function is based on the characteristics of VPVS and the activation function of TNN. VPVS has a main vibration frequency range; it is mixed in two main kinds of noises: trend and random noise. In addition, trend distributes in the low frequency, random noise distributes in the high frequency. The activation function of TNN is continuous and derivable. According to the above characteristics of VPVS and TNN, the proposed thresholding function is as follows:

$$
\eta\left(t_{j, k}, \lambda_{j}\right)= \begin{cases}t_{j, k}-0.5 \lambda_{j}-\frac{0.2 \lambda_{j}}{e^{t_{j, k} / \lambda_{j}-1}}, & t_{j, k} \geq \lambda_{j} \\ 0.3 \lambda_{j}^{-3}\left|t_{j, k}\right|^{4} \operatorname{sgn}\left(t_{j, k}\right), & \left|t_{j, k}\right|<\lambda_{j} \\ t_{j, k}+0.5 \lambda_{j}+\frac{0.2 \lambda_{j}}{e^{-t_{j, k} / \lambda_{j}-1}}, & t_{j, k} \leq-\lambda_{j} .\end{cases}
$$

Our proposed thresholding function is shown in Figure 2, compared with the basic thresholding functions: soft thresholding function (4) and hard thresholding function (5). The coefficients below the threshold of our proposed thresholding function are tuned by a power function rather than set to zero, in order to prevent the useful information loss. Furthermore, its thresholding function and derivative function are continuous to prevent the oscillation and poor smoothness of the reconstructed signal.

The comparison with Zhang's thresholding function (6) and Nasri's thresholding function (7) is shown in Figure 3. Power function is used to tune the coefficients below the threshold, and exponential function is used to tune the coefficients above the threshold; the quantification weight of our proposed thresholding function is more suitable for threshold adjustment with our engineering verification test. 


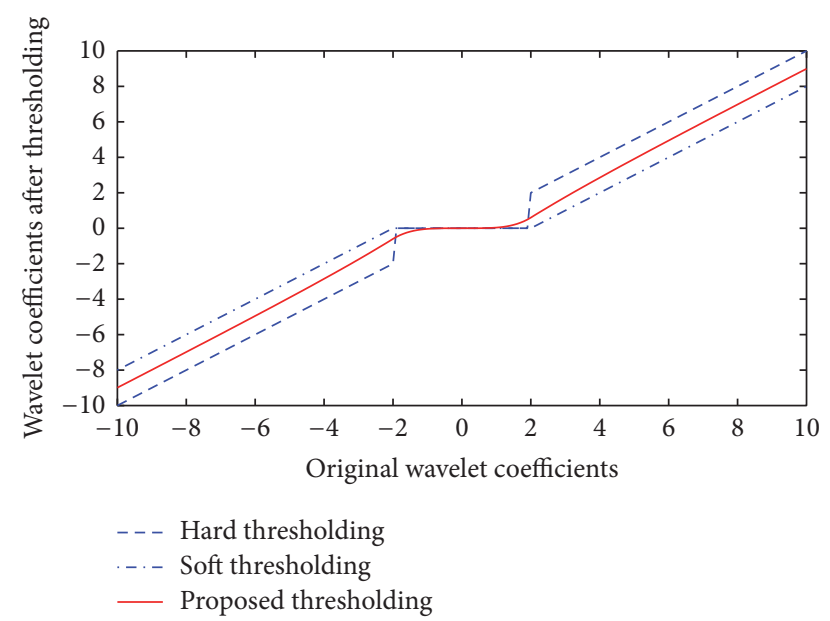

FIGURE 2: Comparison with hard and soft thresholding functions.

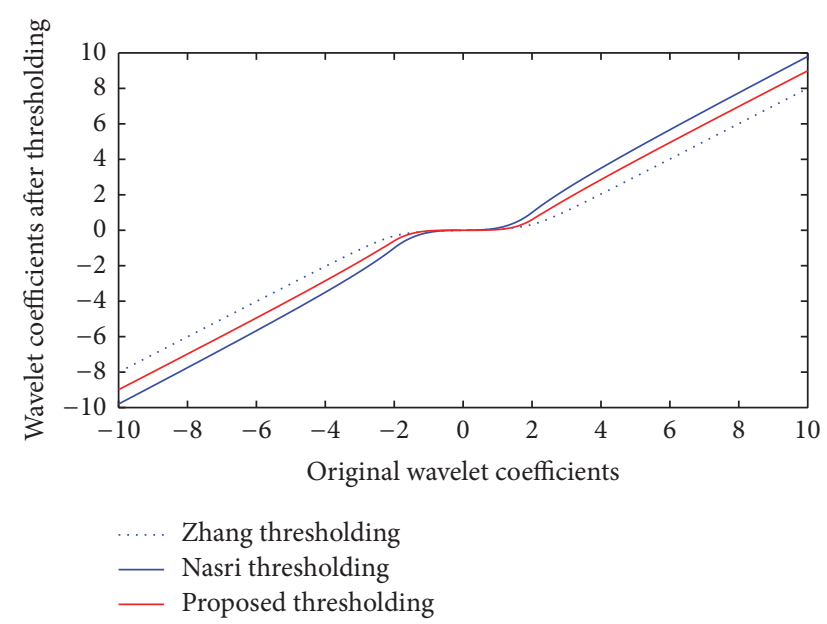

FIGURE 3: Comparison with Zhang's and Nasri's thresholding functions.

Compared with Wang's thresholding function (8) shown in Figure 4, the coefficients below the threshold of our proposed thresholding function are tuned by a power function to prevent the useful information loss, and the derivative of our proposed thresholding function is continuous to prevent the oscillation and poor smoothness of the reconstructed signal.

3.2. The Threshold Selection through Unsupervised Learning of TNN. TNN is based on the neural networks concept and wavelet coefficients thresholding $[11,12]$. Different from the traditional neural networks, the input and output of TNN are the wavelet coefficients of each decomposition level, and the input and output weights are fixed to one; in other words, the wavelet threshold denoising of each decomposition level is independent. The activation function of TNN is the thresholding function, and the incentive factor is the threshold. During the threshold selection process, the wavelet coefficients of each decomposition level are tuned by the thresholding function, and the optimum threshold of each decomposition level is obtained after the learning process.

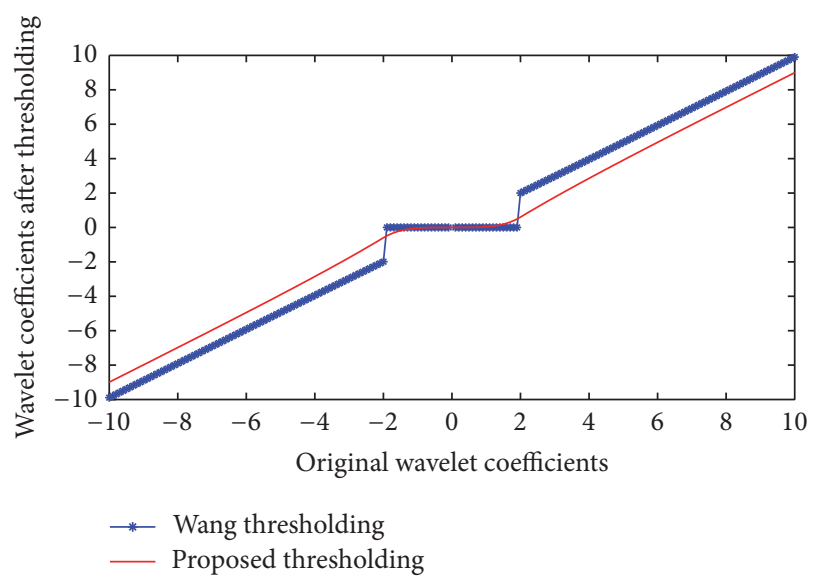

FIGURE 4: Comparison with Wang's thresholding function.

There are two adaptive learning algorithms of TNN toward the optimal threshold: supervised learning and unsupervised learning. Supervised learning is applied to the condition that the original signal or a reference noisy signal is available. In project, it is hard to obtain any available reference signal, so supervised learning cannot be widely used. Unsupervised learning is applied to the condition that only the received noisy signal is known, which is practical in project.

Unsupervised learning is based on the Stein's Unbiased Risk Estimate (SURE) [20]. Suppose the original noisy signal is $s(n)$, the wavelet coefficient of $s(n)$ after the signal reconstruction is $v(x)$, and the wavelet coefficient before the signal reconstruction is $t_{j, k}$; define $g\left(t_{j, k}\right)$ and SURE as follows:

$$
\begin{aligned}
g\left(t_{j, k}\right) & =v\left(t_{j, k}\right)-t_{j, k}, \\
J_{\text {SURE }} & =Z+\left\|g\left(t_{j, k}\right)\right\|^{2}+2 \nabla_{t_{j, k}} \cdot g\left(t_{j, k}\right) \\
& =2 E\left\{\|n\|^{2}\right\}+\left\|g\left(t_{j, k}\right)\right\|^{2}+2 \sum_{k=0}^{M-1} \frac{\partial g_{k}}{\partial t_{j}(k)},
\end{aligned}
$$

where $E\left\{\|n\|^{2}\right\}$ is the noise variance, $M$ is the subband length.

The threshold value $\lambda$ in step $N$ for subband $p$ is calculated as follows:

$$
\begin{aligned}
& \lambda_{p}(N+1)=\lambda_{p}(N)+\Delta \lambda_{p}(N), \\
& \Delta \lambda_{p}(N)=-\alpha \frac{\partial J_{\text {SURE }}}{\partial \lambda} \\
& =-\alpha \frac{\partial}{\partial \lambda}\left(Z+\left\|g\left(t_{j, k}\right)\right\|^{2}+2 \nabla_{t_{j, k}} \cdot g\left(t_{j, k}\right)\right) \\
& =-2 \alpha \sum_{k=0}^{M-1} g_{k} \frac{\partial g_{k}}{\partial \lambda(N)}-2 \alpha \sum_{k=0}^{M-1} \frac{\partial^{2} g_{k}}{\partial t_{j}(k) \partial \lambda(N)},
\end{aligned}
$$

where $\alpha$ is the learning rate.

In the unsupervised learning process, the wavelet coefficients of the original noisy signal $s(n)$ are obtained after wavelet decomposition, and the wavelet coefficients are normalized. Then, the normalized wavelet coefficients are tuned 


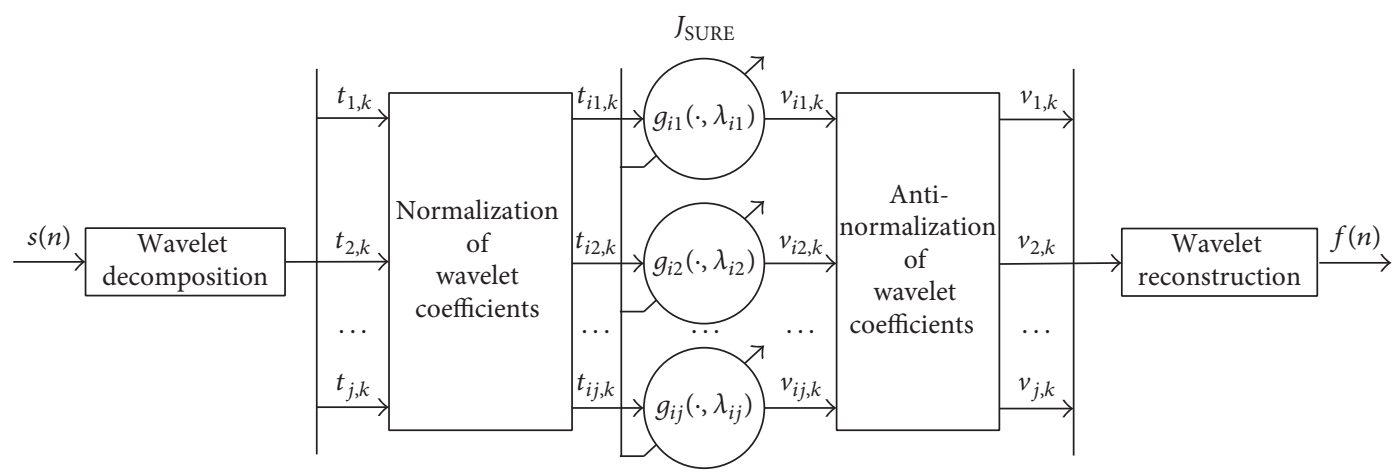

FiguRE 5: TNN structure of unsupervised learning, where $t_{1, k}, t_{2, k}, \ldots, t_{j, k}$ are the wavelet coefficients obtained from the wavelet decomposition of signal $s(n), t_{i 1, k}, t_{i 2, k}, \ldots, t_{i j, k}$ are the normalized wavelet coefficients, $v_{i 1, k}, v_{i 2, k}, \ldots, v_{i j, k}$ are the new normalized wavelet coefficients tuned by thresholding function (11), and the optimal thresholds of thresholding function (11) are selected by (12) and (13), and $v_{1, k}, v_{2, k}, \ldots, v_{j, k}$ are the antinormalized wavelet coefficients, which is used for the wavelet reconstruction of signal $f(n)$.

by thresholding function (11), and the optimal thresholds of thresholding function (11) are selected by (12) and (13). After the optimal thresholds are obtained, the new wavelet coefficients are obtained and antinormalized. Finally, the antinormalized wavelet coefficients are reconstructed to build the denoised signal $f(n)$. The process is depicted in Figure 5 .

\section{VPVS Models and Analysis of Power Spectral and Energy Distribution}

4.1. The Establishment of VPVS Models. The establishment of VPVS models is based on the VPVS characteristics, which include two aspects: the characteristics of original VPVS and noise. The original VPVS can be speculated from the vibration sources of vehicle platform. The vibration sources of vehicle platform include crew-induced vibration and the vibration transmitted from engine and generator. The frequency range of crew-induced vibration is approximately $1 \sim 3 \mathrm{~Hz}$, and the frequency range of engine and generator vibration is approximately $6 \sim 65 \mathrm{~Hz}[21]$. Furthermore, the main vibration source of vehicle platform is the vibration transmitted from engine and generator, and its vibration frequency range is $6 \sim 65 \mathrm{~Hz}$ in project.

As for the noise characteristics, there are two main components of noises: trend and random noise. The main sources of trend include three parts: zero drift caused by temperature change of test equipment, instability of low-frequency performance outside the frequency range of accelerometer, and environmental interference around the accelerometer, which makes original VPVS deviate from the baseline. The main source of random noise is the random interference during the sampling process of data collector, which superimposes a wideband random noise on the original VPVS [22].

According to the above theoretical analysis of VPVS characteristics, the VPVS models consist of three parts: the original VPVS, trend, and random noise. The original VPVS has two main features: the dominant frequency and the main frequency range. According to the two main features, two original VPVS models are established; one is a sine signal with dominant frequency, and the other is sweep signal with the main frequency range. The denoising method can be proved, if the two original VPVS models are retained relatively complete after the denoising method. The trend is associated with the inherent test conditions, which can be collected in the condition that the engine and generator are inoperative. The random noise has similar characteristics with Gaussian white noise and can be expressed by Gaussian white noise. Suppose the VPVS model $s(n)$ given by

$$
s(n)=x(n)+e_{1}(n)+e_{2}(n),
$$

where $x(n)$ is the original VPVS model, $e_{1}(n)$ is the trend collected in the test, and $e_{2}(n)$ is the Gaussian white noise; its variance is selected as 0.3 , according to the estimated noise levels in the test.

In the practical vehicle platform vibration test, the sampling frequency is set to $1 \mathrm{kHz}$. In order to clarify the signal details clearly, the number of sampling points is selected as 3000. The dominant vibration frequency is $25 \mathrm{~Hz}$, and the main vibration frequency range is $6 \sim 65 \mathrm{~Hz}$. The original sine VPVS model $x_{1}(n)$ is defined as follows:

$$
x_{1}(n)=A_{1} \sin 2 \pi f_{1} n,
$$

where $f_{1}$ is the dominant vibration frequency and $A_{1}$ is the amplitude of the original sine VPVS model, which is selected as 1.5, according to the estimated signal magnitude in the test.

The original sine VPVS model $x_{1}(n)$ and the sine VPVS model $s_{1}(n)$ are shown in Figure 6. The SNR is $4.96 \mathrm{~dB}$, and RMSE is $0.08 \mathrm{~m} / \mathrm{s}^{2}$.

The original sweep VPVS model $x_{2}(n)$ is defined as follows:

$$
x_{2}(n)=A_{2} \cos \left(2 \pi f_{i}(n) n\right),
$$

where $A_{2}$ is the amplitude of the original sweep VPVS model, which is selected as 1.5. The main vibration frequency range $f_{i}(n)$ is as follows:

$$
f_{i}(n)=f_{0}+\frac{f_{1}-f_{0}}{t_{s}} n
$$

where $f_{0}$ is the initial frequency, $f_{1}$ is the target frequency. $t_{s}$ is calculated as 3 , according to the sampling frequency and sampling points. 

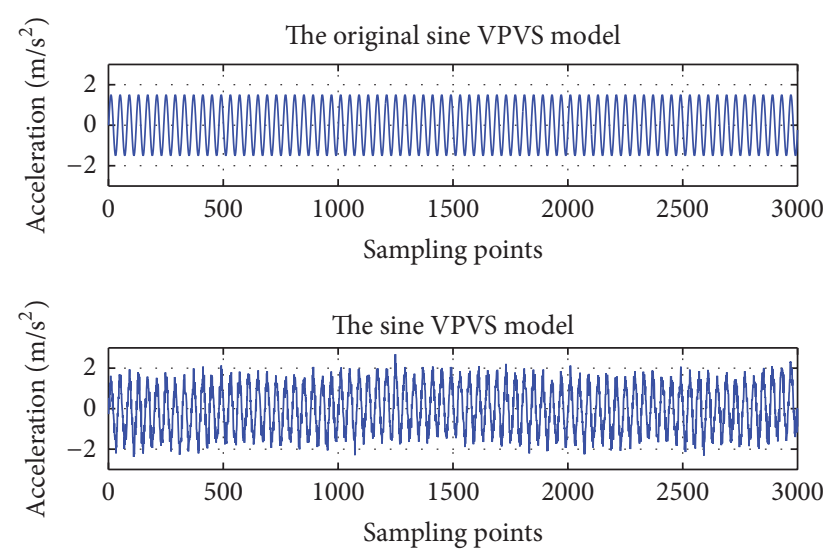

FIGURE 6: The original sine VPVS model and sine VPVS model.
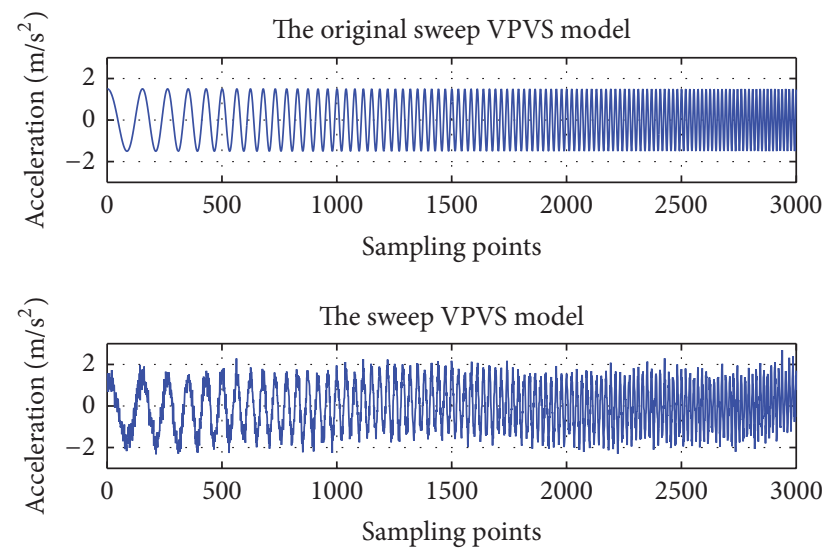

FIGURE 7: The original sweep VPVS model and sine VPVS model.

The original sweep VPVS model $x_{2}(n)$ and the sweep VPVS model $s_{2}(n)$ are shown in Figure 7. The SNR is $4.96 \mathrm{~dB}$, and RMSE is $0.08 \mathrm{~m} / \mathrm{s}^{2}$.

4.2. Power Spectral and Energy Distribution of VPVS Models. The analysis of power spectral and energy distribution is essential to design the wavelet denoising algorithm flow. The power spectral distribution of Gaussian white noise is uniform distribution [23]. The power spectral distribution of trend collected in the test is shown in Figure 8, which mainly distributes in the low-frequency range lower than $6 \mathrm{~Hz}$.

The models of energy distribution analysis include six parts: trend, Gaussian white noise, the original sine VPVS model $x_{1}(n)$, the sine VPVS model $s_{1}(n)$, the original sweep VPVS model $x_{2}(n)$, and the sweep VPVS model $s_{2}(n)$. The energy of each part is calculated as follows [24]:

$$
E(j)=\sum_{k \in z} t_{j, k}^{2}
$$

where $E(j)$ is the energy of the wavelet coefficients $t_{j, k}$ in the decomposition level $j$ and $t_{j, k}$ includes the approximate coefficients $c_{3}$ and the detail coefficients $d_{1}, d_{2}$, and $d_{3}$.

The energy distribution of each parts is shown in Figure 9. It shows that the energy of the original VPVS models mainly
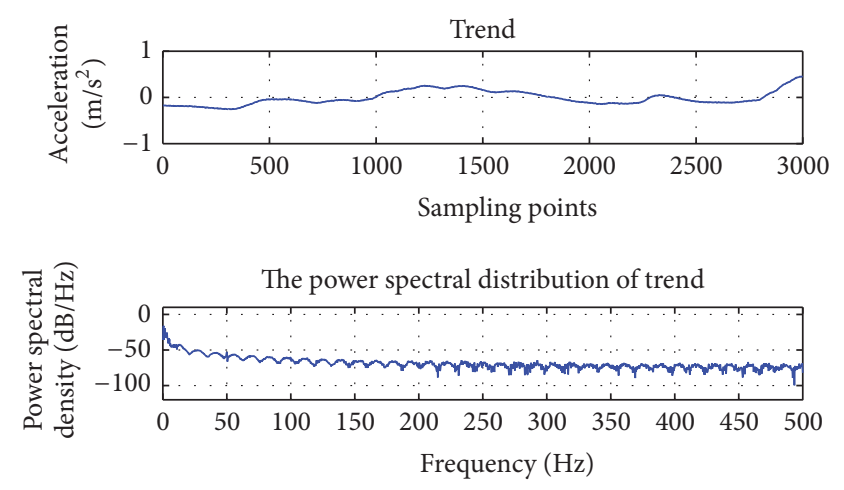

FIGURE 8: The power spectral distribution of trend.

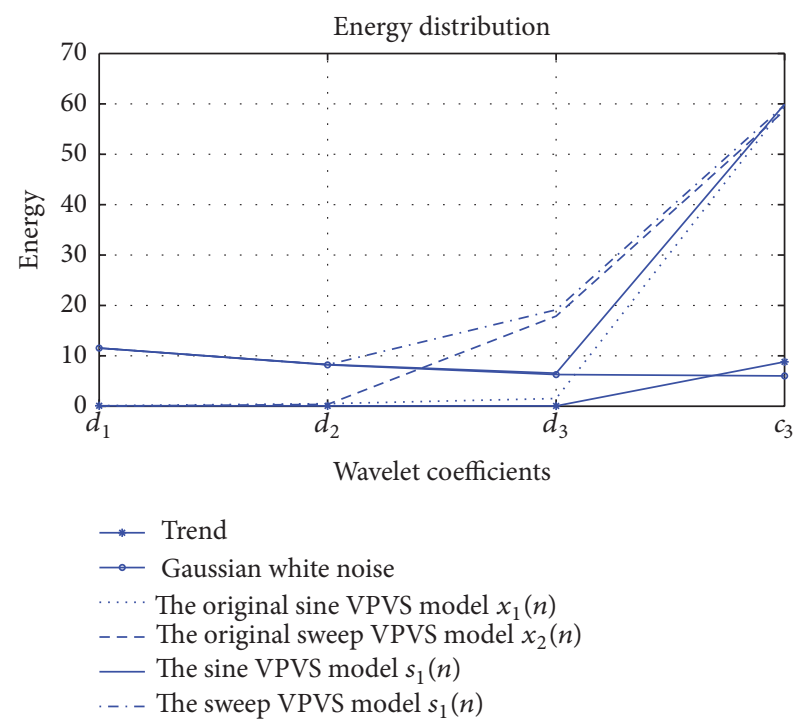

FIGURE 9: The energy distribution of VPVS model.

distributes in the approximate coefficients $c_{3}$ and the detail coefficients $d_{3}$. However, the energy of the VPVS models distributes in all the wavelet coefficients, which is influenced by the trend and Gaussian white noise. The energy of trend mainly distributes in the approximate coefficients $c_{3}$. The energy of Gaussian white noise distributes in the all the wavelet coefficients and decreases with the increasing decomposition level.

In conclusion, the wavelet denoising algorithm flow can be divided into two parts: eliminating the influence of trend on the approximate coefficients and eliminating the influence of Gaussian white noise on the detail coefficients.

\section{Wavelet Denoising of VPVS Models}

5.1. VPVS Denoising Flow. The VPVS denoising flow is proposed to denoise VPVS polluted by the trend and Gaussian white noise, which is depicted in Figure 10.

Firstly, the VPVS $s(n)$ is filtered into two parts: the highfrequency VPVS $s_{h}(n)$ and the low-frequency VPVS $s_{l}(n)$. Each part is decomposed and reconstructed, respectively, the detail coefficients of both parts are processed by unsupervised 


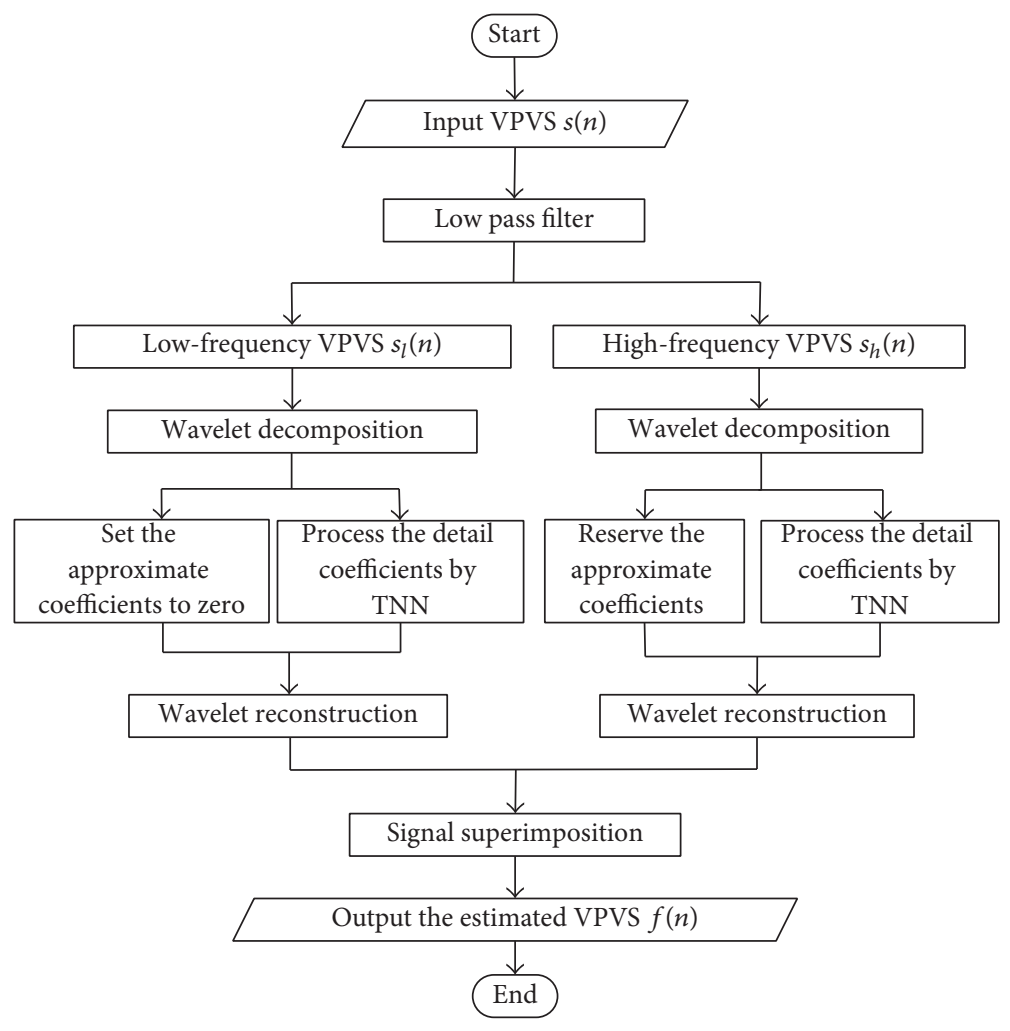

FIGURE 10: Flow chart of proposed VPVS denoising method.

learning of TNN, the approximate coefficients of $s_{h}(n)$ are reserved, and the approximate coefficients of $s_{l}(n)$ are set to zero; the denoised signal $f(n)$ is obtained through the superposition of denoised $s_{h}(n)$ and $s_{l}(n)$.

5.2. Simulation of VPVS Denoising Method. Six denoising approaches are used for VPVS denoising. These are Donoho's soft and hard thresholding method, the denoising methods mentioned in the publications [11-13], and the VPVS denoising method proposed in this paper.

According to the theoretical background mentioned in Section 2.1, the wavelet basis function is db14; the maximum decomposition level is 3. According to the multiresolution threshold method [25], the initial threshold $\lambda_{j}$ of unsupervised learning is set as follows:

$$
\lambda_{j}=\sigma \frac{\sqrt{2 \ln \left(\text { length }\left(d_{i, k}\right)\right)}}{\ln (j+1)},
$$

where the noise variance estimation $\sigma$ is calculated as follows:

$$
\sigma=\frac{\operatorname{median}\left(\left|d_{i, k}\right|\right)}{0.6754} .
$$

In this paper, the thresholds of soft thresholding method and hard thresholding method are set as functions (19) and (20). The initial threshold of TNN method is set as functions (19) and (20), and the threshold is selected by functions (12) and (13). In addition, the VPVS denoising flow is applied in proposed thresholding method.
TABLE 1: The optimal threshold in proposed thresholding method.

\begin{tabular}{lcc}
\hline & Decomposition level & Optimal threshold \\
\hline \multirow{2}{*}{ Low-frequency sine } & 1 & 1.51 \\
VPVS & 2 & 1.30 \\
& 3 & 1.21 \\
\hline \multirow{2}{*}{ High-frequency sine } & 1 & 2.57 \\
VPVS & 2 & 2.17 \\
& 3 & 1.75 \\
\hline \multirow{2}{*}{ Low-frequency sweep } & 1 & 1.78 \\
VPVS & 2 & 1.75 \\
& 3 & 1.58 \\
\hline \multirow{2}{*}{ High-frequency } & 1 & 2.71 \\
sweep VPVS & 2 & 2.19 \\
& 3 & 1.90 \\
\hline
\end{tabular}

Table 1 shows the optimal threshold selected by the unsupervised learning of TNN in proposed thresholding method, and the learning termination condition is that the ratio of $\Delta \lambda(N)$ and $\lambda(N)$ is less than $10^{-6}$.

For sine VPVS model, the SNR and RMSE are shown in Table 2, which is calculated by functions (9) and (10). In Table 2, compared with other denoising methods, the proposed thresholding method brings improvement to the indexes of SNR and RMSE. The SNR increases by about $30 \%$, and the RMSE decreases by about $17 \%$. 

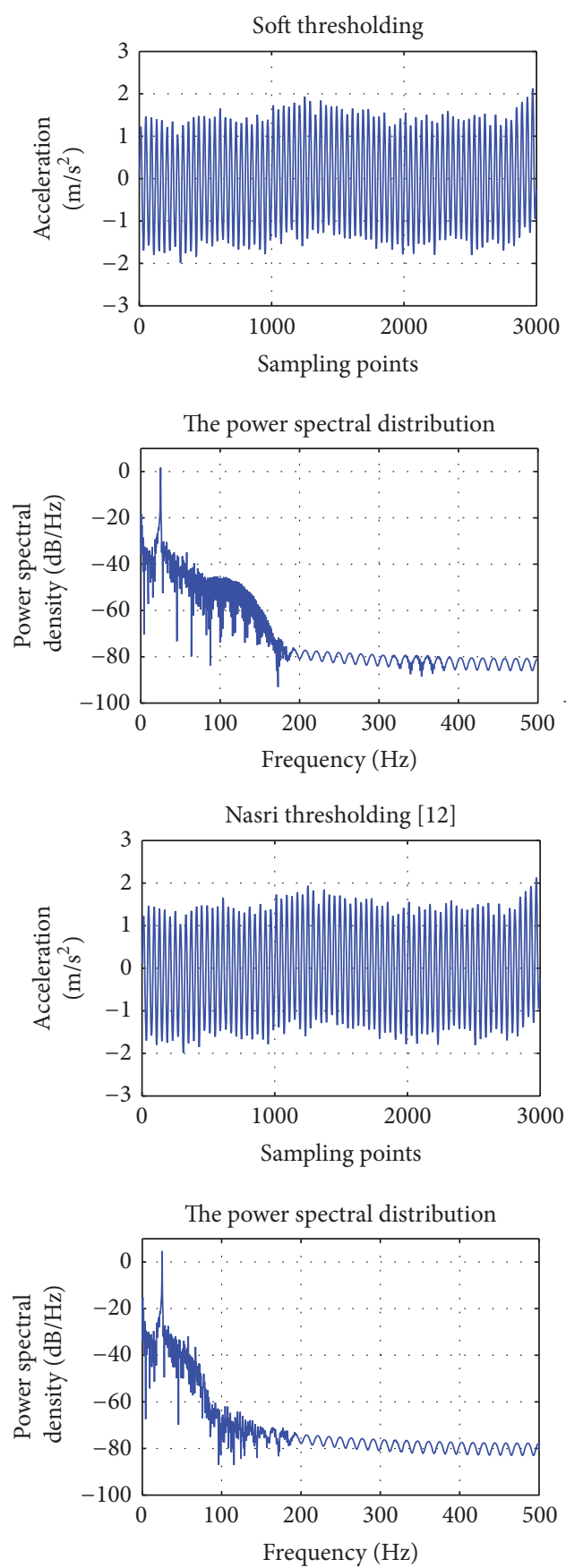
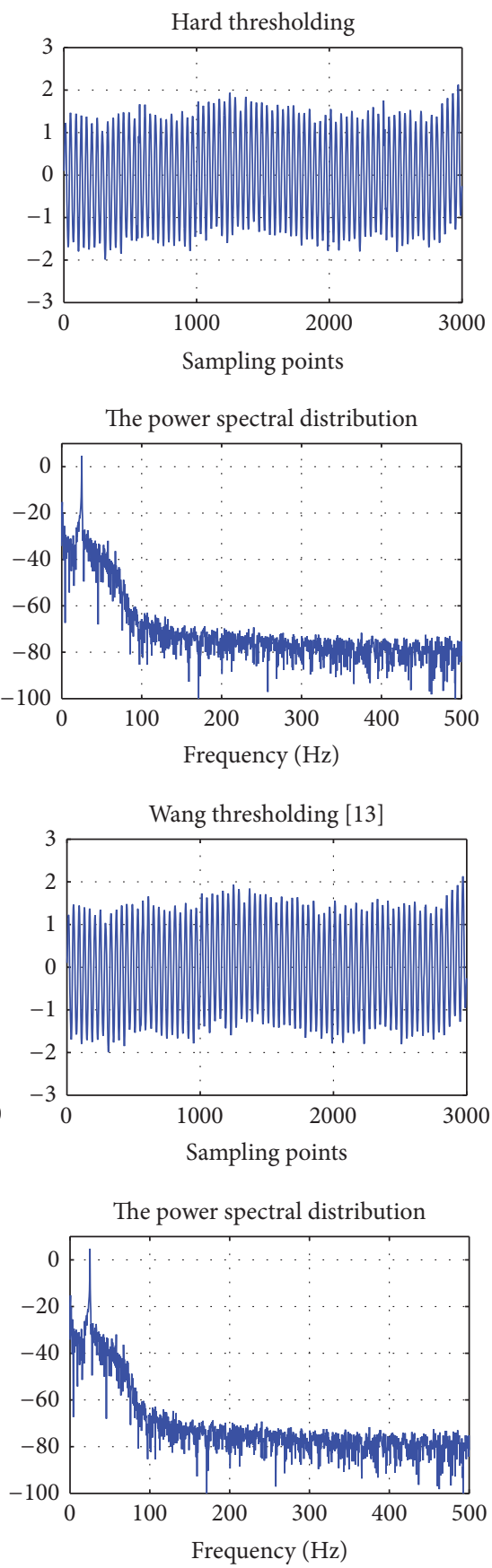
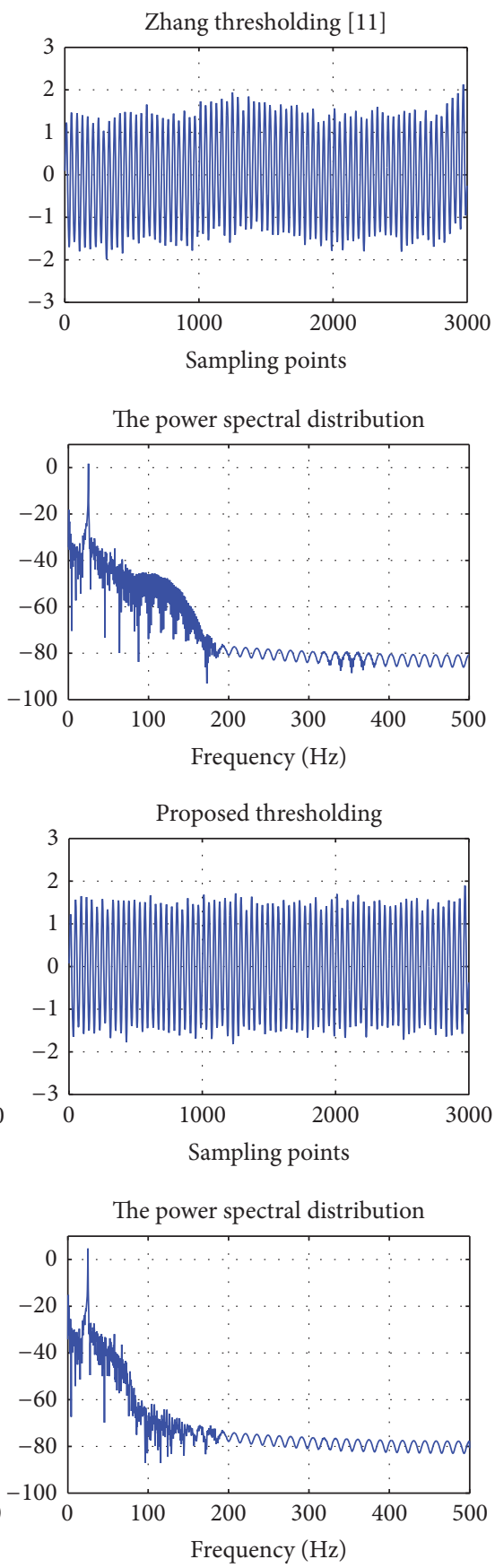

FIGURE 11: The denoising results of sine VPVS model.

The denoising results of sine VPVS model are shown in Figure 11. According to Figure 11, for time-domain analysis, the profile of the sine VPVS model denoised by the proposed thresholding method is closer to the profile of original sine VPVS model. For frequency-domain analysis, the main frequency reserves better at $25 \mathrm{~Hz}$; the low-frequency noise lower than $25 \mathrm{~Hz}$ and the high-frequency noise higher than $25 \mathrm{~Hz}$ are attenuated more adequately. The proposed denoising method is efficient for denoising sine VPVS polluted by the trend and Gaussian white noise.
For sweep VPVS model, the SNR and RMSE are shown in Table 3, which is calculated by functions (9) and (10).

In Table 3, compared with other denoising methods, the proposed thresholding method brings improvement to the indexes of SNR and RMSE. The SNR increases by about $20 \%$ and the RMSE decreases by about $17 \%$.

The denoising results of sweep VPVS model are shown in Figure 12. According to Figure 12, for time-domain analysis, the profile of the sweep VPVS model denoised by the proposed thresholding method is closer to the profile of 

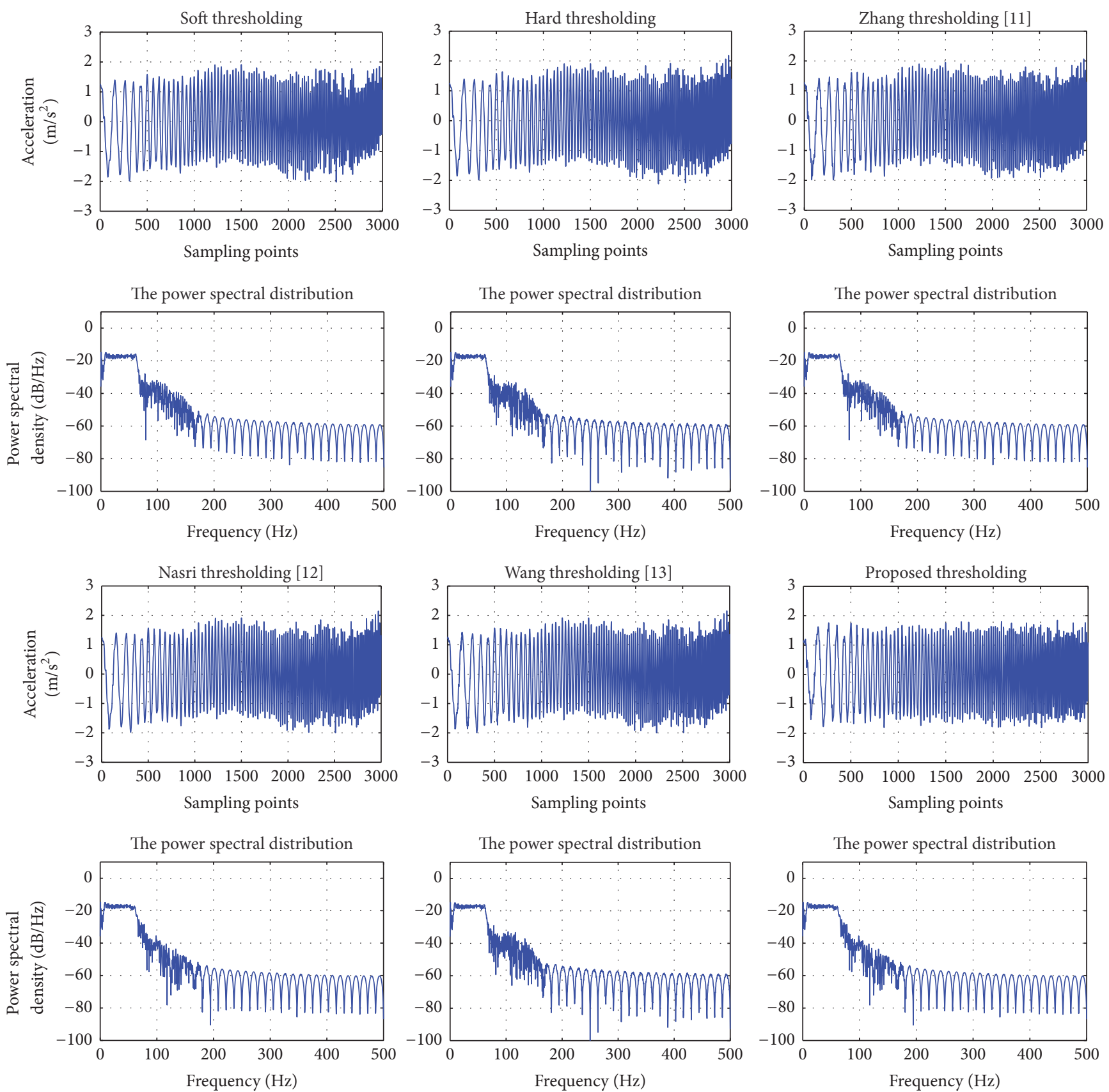

FIGURE 12: The denoising results of sweep VPVS model.

original sweep VPVS model. For frequency-domain analysis, the main frequency range reserves better from 6 to $65 \mathrm{~Hz}$; the low-frequency noise lower than $6 \mathrm{~Hz}$ and the high-frequency noise higher than $65 \mathrm{~Hz}$ are attenuated more adequately. The proposed denoising method is efficient for denoising sweep VPVS polluted by the trend and Gaussian white noise.

In conclusion, the proposed denoising method is efficient for denoising both sine and sweep VPVS model polluted by the trend and Gaussian white noise and performs better in the smoothness and integrity of both sine and sweep VPVS model.

\section{Vehicle Platform Vibration Test and Results Analysis}

The apparatus of vehicle platform vibration test is shown in Figure 13. It consists of three main parts: signal measurement section, signal collection section, and vehicle equipment. The signal measurement section is the piezoelectric accelerometer of model 356A26PCB. The signal collection section includes a data collector and a laptop. The vehicle equipment includes a precise optical measuring instrument and a vehicle platform.

In the vehicle platform vibration test, the accelerometer is placed in the sensitive vibration position, and the sensitive 


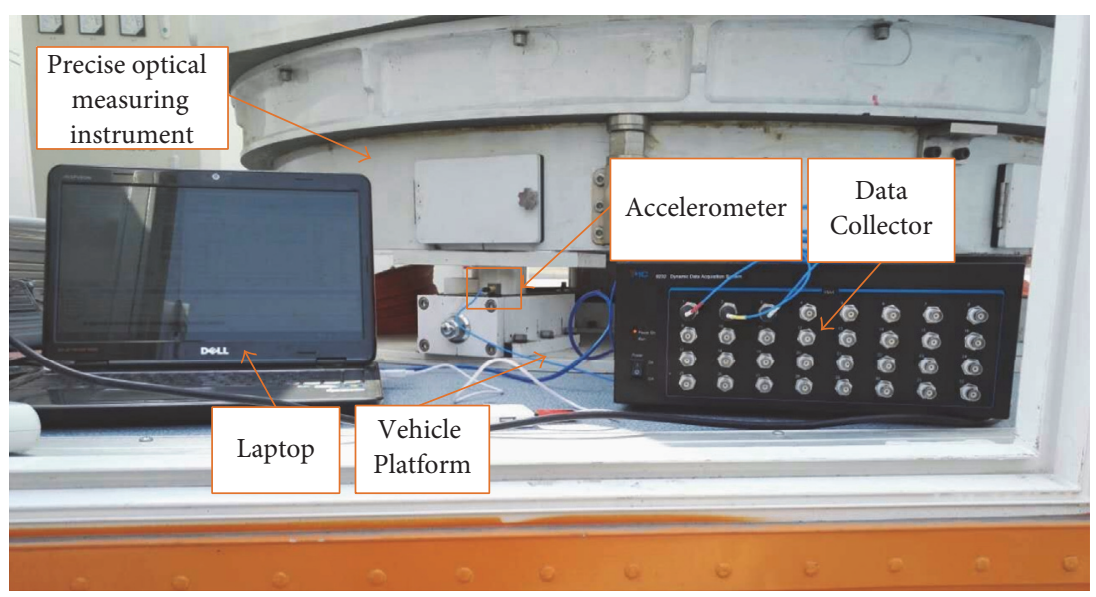

FIGURE 13: The apparatus of vehicle platform vibration test.

TABLE 2: The SNR and RMSE results of denoising methods.

\begin{tabular}{lcc}
\hline & \multicolumn{2}{c}{ Sine VPVS model } \\
& SNR $(\mathrm{dB})$ & RMSE $\left(\mathrm{m} / \mathrm{s}^{2}\right)$ \\
\hline $\begin{array}{l}\text { Soft thresholding } \\
\text { Hard thresholding }\end{array}$ & 7.50 & 0.06 \\
$\begin{array}{l}\text { Zhang's } \\
\text { thresholding [11] }\end{array}$ & 7.51 & 0.06 \\
$\begin{array}{l}\text { Nasri's } \\
\text { thresholding [12] }\end{array}$ & 7.52 & 0.06 \\
$\begin{array}{l}\text { Wang's } \\
\text { thresholding [13] }\end{array}$ & 7.46 & 0.06 \\
$\begin{array}{l}\text { Proposed } \\
\text { thresholding }\end{array}$ & 9.76 & 0.06 \\
\hline
\end{tabular}

TABLE 3: The SNR and RMSE results of denoising methods.

\begin{tabular}{lcc}
\hline & \multicolumn{2}{c}{ Sweep VPVS model } \\
& SNR $(\mathrm{dB})$ & RMSE $\left(\mathrm{m} / \mathrm{s}^{2}\right)$ \\
\hline Soft thresholding & 6.51 & 0.07 \\
$\begin{array}{l}\text { Hard thresholding } \\
\text { Zhang's }\end{array}$ & 6.99 & 0.06 \\
thresholding [11] & 7.00 & 0.06 \\
$\begin{array}{l}\text { Nasri's } \\
\text { thresholding [12] }\end{array}$ & 7.09 & 0.06 \\
$\begin{array}{l}\text { Wang's } \\
\text { thresholding [13] }\end{array}$ & 7.06 & 0.06 \\
$\begin{array}{l}\text { Proposed } \\
\text { thresholding }\end{array}$ & 8.39 & 0.05 \\
\hline
\end{tabular}

axis of the accelerometer is perpendicular to the measuring plane. The sampling frequency is set to $1 \mathrm{kHz}$. In order to clarify the signal details clearly, the number of sampling points is selected as 3000. The power spectral analysis of the measured signal is shown in Figure 14.

The main frequency range of measured signal is from 10 to $60 \mathrm{~Hz}$, which is included in the theoretical range $6 \sim 65 \mathrm{~Hz}$.
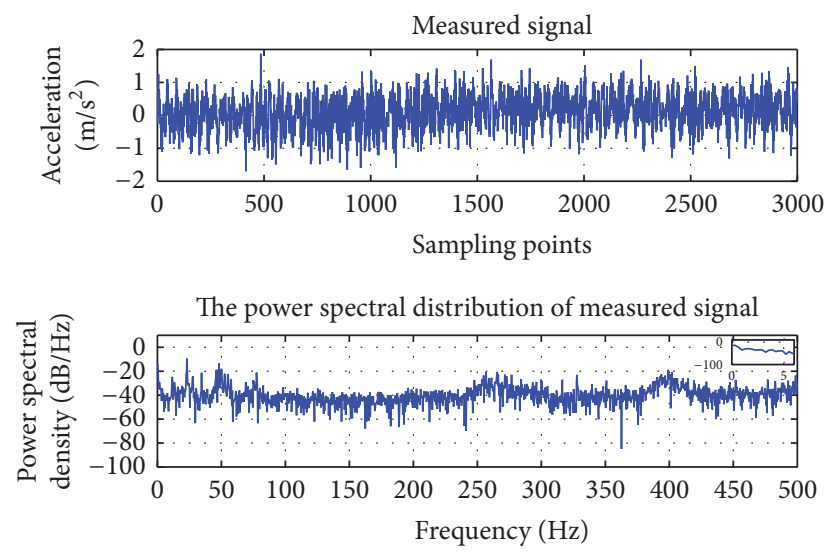

FIGURE 14: The power spectral analysis of VPVS.

The power spectral distribution of low-frequency signal lower than $6 \mathrm{~Hz}$ is from -10 to $-50 \mathrm{~dB}$, and the high-frequency signal higher than $65 \mathrm{~Hz}$ is from -20 to $-60 \mathrm{~dB}$. All denoising methods mentioned above are applied to the measured signal; the result is shown in Figure 15.

The components of the measured signal are unknown, so the SNR and RMSE of the measured signal cannot be calculated. However, the frequency component can be the evaluation criterion instead according to the characteristics of the VPVS. Compared with the measured signal in Figure 15, the signal denoised by the proposed thresholding method has some superior characteristics.

For one thing, the main frequency range reserves from 10 to $60 \mathrm{~Hz}$, the low-frequency signal lower than $6 \mathrm{~Hz}$ attenuates from $-10 \sim-50 \mathrm{~dB}$ to $-40 \sim-60 \mathrm{~dB}$ in our proposed thresholding method, but the attenuation at these frequencies is not obvious from the enlarged view in other thresholding methods. For another, the high-frequency signal higher than $65 \mathrm{~Hz}$ attenuates from $-20 \sim-60 \mathrm{~dB}$ to less than $-60 \mathrm{~dB}$ in our proposed thresholding method, which attenuates more thoroughly from the power spectral distribution, compared with other thresholding methods. 

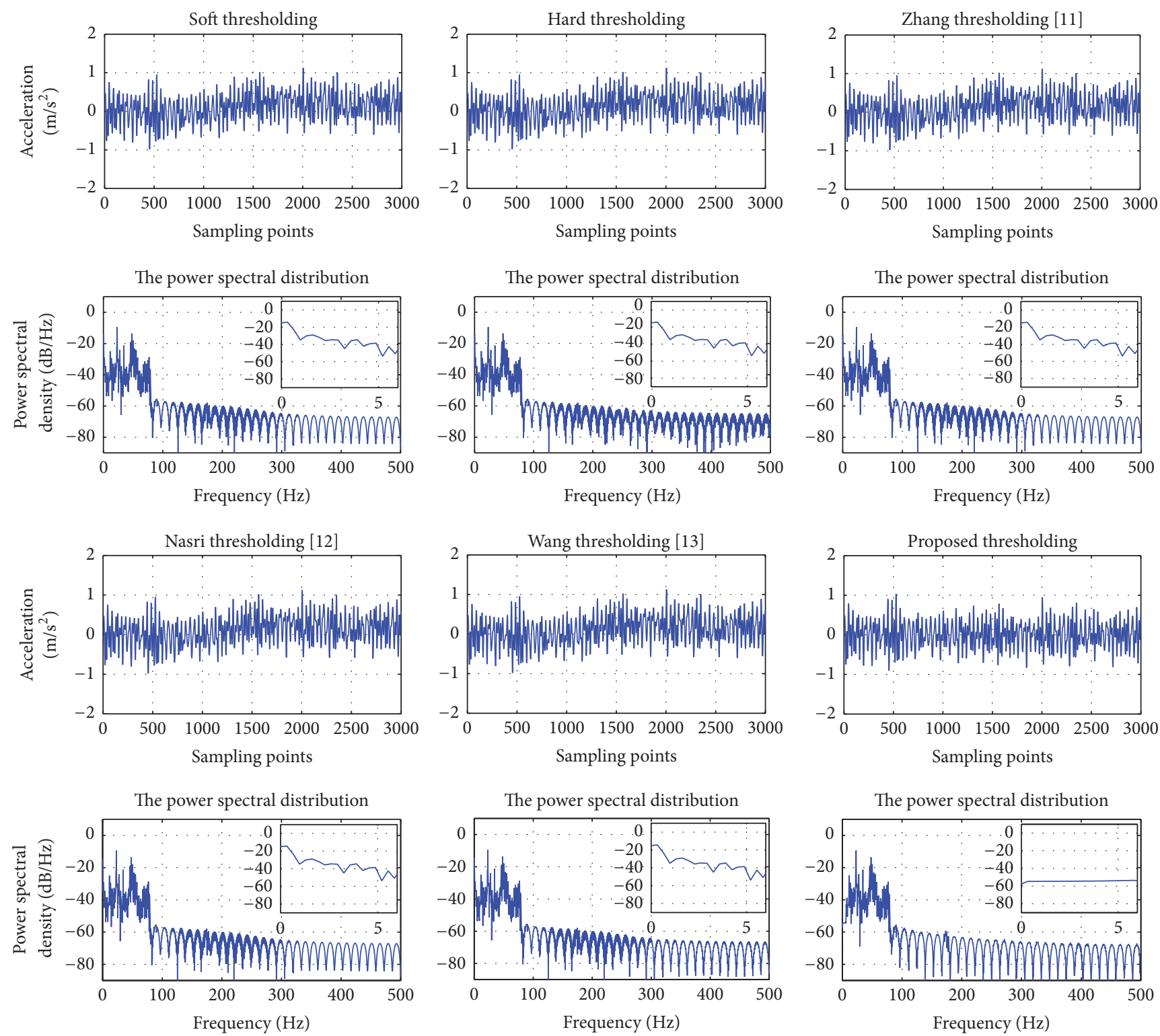

FIGURE 15: The denoised results of VPVS.

In conclusion, compared with other thresholding methods, the proposed thresholding method performs better in both low-frequency part and high-frequency part and reserves the VPVS more completely. The results show that the proposed thresholding method is efficient for denoising VPVS polluted by the trend and random noise.

\section{Conclusion}

In this paper, a VPVS denoising method is presented. A thresholding function adaptive to VPVS and TNN is established, and the threshold is selected by TNN. According to the characteristics of VPVS, two signal models mixed in trend and random noise are constructed as VPVS models. A VPVS denoising flow is proposed based on the power spectral and energy distribution of the VPVS models.
This paper mainly focuses on three points: the thresholding function adaptive to VPVS and TNN, the application of TNN in VPVS denoising field, and the two signal models mixed in trend and random noise. The simulation results show that it brings improvement to the indexes of SNR and RMSE. The experiment demonstrates that it is efficient for denoising VPVS polluted by the trend and random noise.

In the future, the rapidness of the proposed VPVS denoising method should be considered, so that the method can be portable to hardware platform to achieve real-time denoising and larger applications.

\section{Competing Interests}

The authors declare that they have no competing interests. 


\section{Acknowledgments}

This work is supported by the Jilin Province Key Scientific and Technological Projects of China (20150204013GX) and the Jilin Province Key Scientific and Technological Projects of China (20160204053GX).

\section{References}

[1] D. L. Donoho, "De-noising by soft-thresholding," Institute of Electrical and Electronics Engineers. Transactions on Information Theory, vol. 41, no. 3, pp. 613-627, 1995.

[2] J. Li, C. Cheng, T. Jiang, and S. Grzybowski, "Wavelet denoising of partial discharge signals based on genetic adaptive threshold estimation," IEEE Transactions on Dielectrics and Electrical Insulation, vol. 19, no. 2, pp. 543-549, 2012.

[3] B. Gu, X. Sun, and V. S. Sheng, "Structural minimax probability machine," IEEE Transactions on Neural Networks and Learning Systems, 2016.

[4] B. Gu, V. S. Sheng, K. Y. Tay, W. Romano, and S. Li, "Incremental support vector learning for ordinal regression," IEEE Transactions on Neural Networks and Learning Systems, vol. 26, no. 7, pp. 1403-1416, 2015.

[5] B. Gu and V. S. Sheng, "A robust regularization path algorithm for $\nu$-support vector classification," IEEE Transactions on Neural Networks and Learning Systems, pp. 1-8, 2016.

[6] Z. Xia, X. Wang, X. Sun, Q. Liu, and N. Xiong, "Steganalysis of LSB matching using differences between nonadjacent pixels," Multimedia Tools and Applications, vol. 75, no. 4, pp. 1947-1962, 2016.

[7] X. Wen, L. Shao, Y. Xue, and W. Fang, "A rapid learning algorithm for vehicle classification," Information Sciences, vol. 295, no. 1, pp. 395-406, 2015.

[8] W. Deng, H. Zhao, J. Liu et al., "An improved CACO algorithm based on adaptive method and multi-variant strategies," Soft Computing, vol. 19, no. 3, pp. 701-713, 2014.

[9] W. Deng, R. Chen, B. He, Y. Q. Liu, L. F. Yin, and J. H. Guo, "A novel two-stage hybrid swarm intelligence optimization algorithm and application," Soft Computing, vol. 16, no. 10, pp. 1707-1722, 2012.

[10] Y. Zheng, B. Jeon, D. Xu, Q. M. J. Wu, and H. Zhang, "Image segmentation by generalized hierarchical fuzzy C-means algorithm," Journal of Intelligent and Fuzzy Systems, vol. 28, no. 2, pp. 961-973, 2015.

[11] X.-P. Zhang, "Thresholding neural network for adaptive noise reduction," IEEE Transactions on Neural Networks, vol. 12, no. 3, pp. 567-584, 2001.

[12] M. Nasri and H. Nezamabadi-pour, "Image denoising in the wavelet domain using a new adaptive thresholding function," Neurocomputing, vol. 72, no. 4-6, pp. 1012-1025, 2009.

[13] X. Wang, X. Ou, B.-W. Chen, and M. Kim, "Image denoising based on improved wavelet threshold function for wireless camera networks and transmissions," International Journal of Distributed Sensor Networks, vol. 2015, Article ID 670216, 23 pages, 2015.

[14] T.-W. Yang, P.-F. Chen, J.-D. Han, and W.-L. Xu, "Wavelet transform analysis of optic fiber force sensing signals at puncture needle tip," Optics and Precision Engineering, vol. 23, no. 8, pp. 2149-2157, 2015.

[15] R. Yan, R. X. Gao, and X. Chen, "Wavelets for fault diagnosis of rotary machines: a review with applications," Signal Processing, vol. 96, pp. 1-15, 2014.
[16] Y.-P. Zang, D.-J. Zhang, and W.-Z. Wang, "Per-level threshold de-noising method using wavelet and its application in engine vibration analysis," Journal of Vibration and Shock, vol. 28, no. 8, pp. 57-60, 2009.

[17] R. Yang and M. Ren, "Wavelet denoising using principal component analysis," Expert Systems with Applications, vol. 38, no. 1, pp. 1073-1076, 2011.

[18] L. Jing-Yi, L. Hong, Y. Dong, and Z. Yan-Sheng, "A new wavelet threshold function and denoising application," Mathematical Problems in Engineering, vol. 2016, Article ID 3195492, 8 pages, 2016.

[19] Z. Pan, Y. Zhang, and S. Kwong, "Efficient motion and disparity estimation optimization for low complexity multiview video coding," IEEE Transactions on Broadcasting, vol. 61, no. 2, pp. 166-176, 2015.

[20] C. M. Stein, "Estimation of the mean of a multivariate normal distribution," The Annals of Statistics, vol. 9, no. 6, pp. 1135-1151, 1981.

[21] C. L. Zhang, Study on Active Vibration Control of Micro-Manufacturing Platform, Zhejing University, Hangzhou, China, 2003.

[22] X. Hu and J. Wang, The Application of MATLAB in Signal Processing, China Water Power Press, Beijing, China, 2006.

[23] J. Li, T. Jiang, S. Grzybowski, and C. Cheng, "Scale dependent wavelet selection for de-noising of partial discharge detection," IEEE Transactions on Dielectrics and Electrical Insulation, vol. 17, no. 6, pp. 1705-1714, 2010.

[24] W.-Y. Dong, H. Ding, X.-S. Dong, and Y.-F. Wang, "An adaptive wavelet threshold de-nosing both in low and high frequency domains," Acta Electronica Sinica, vol. 43, no. 12, pp. 2374-2380, 2015.

[25] D. L. Donoho and I. M. Johnstone, "Adapting to unknown smoothness via wavelet shrinkage," Journal of the American Statistical Association, vol. 90, no. 432, pp. 1200-1224, 1995. 


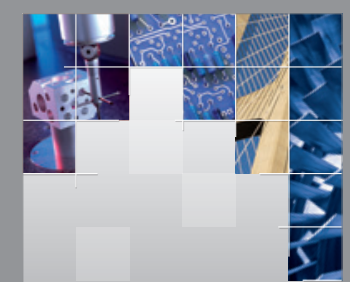

\section{Enfincering}
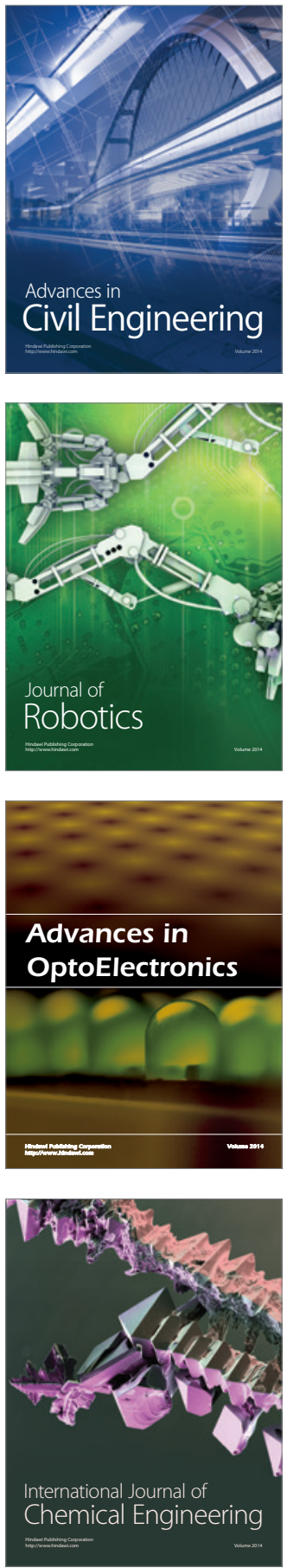

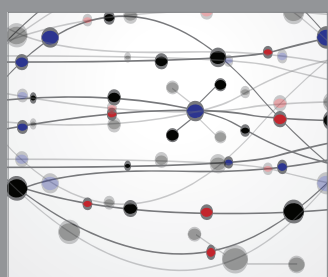

The Scientific World Journal

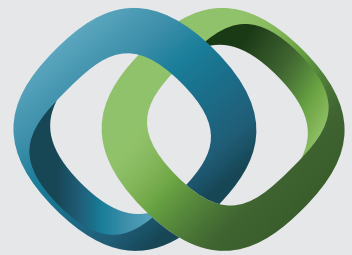

\section{Hindawi}

Submit your manuscripts at

https://www.hindawi.com
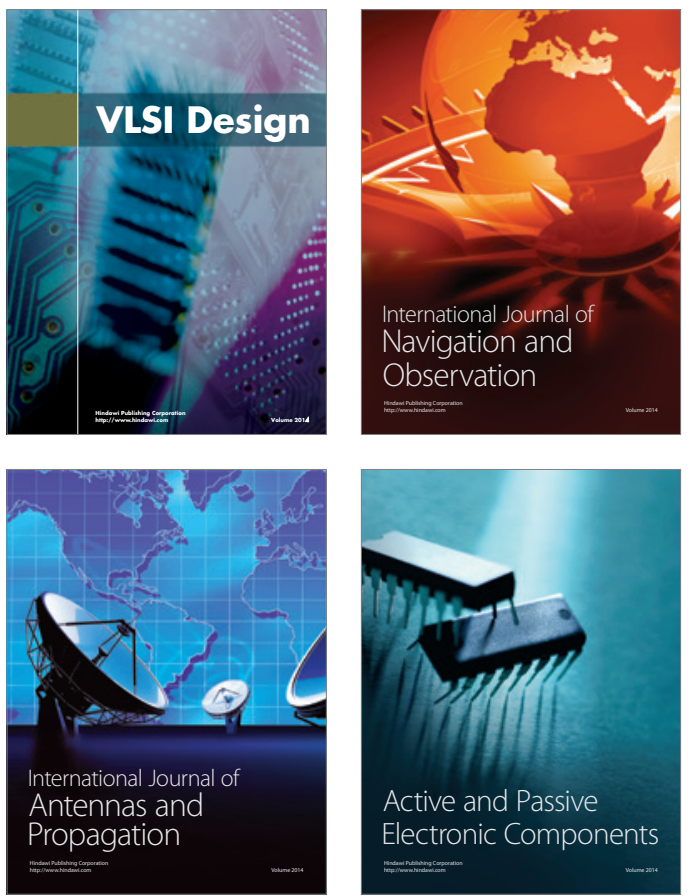
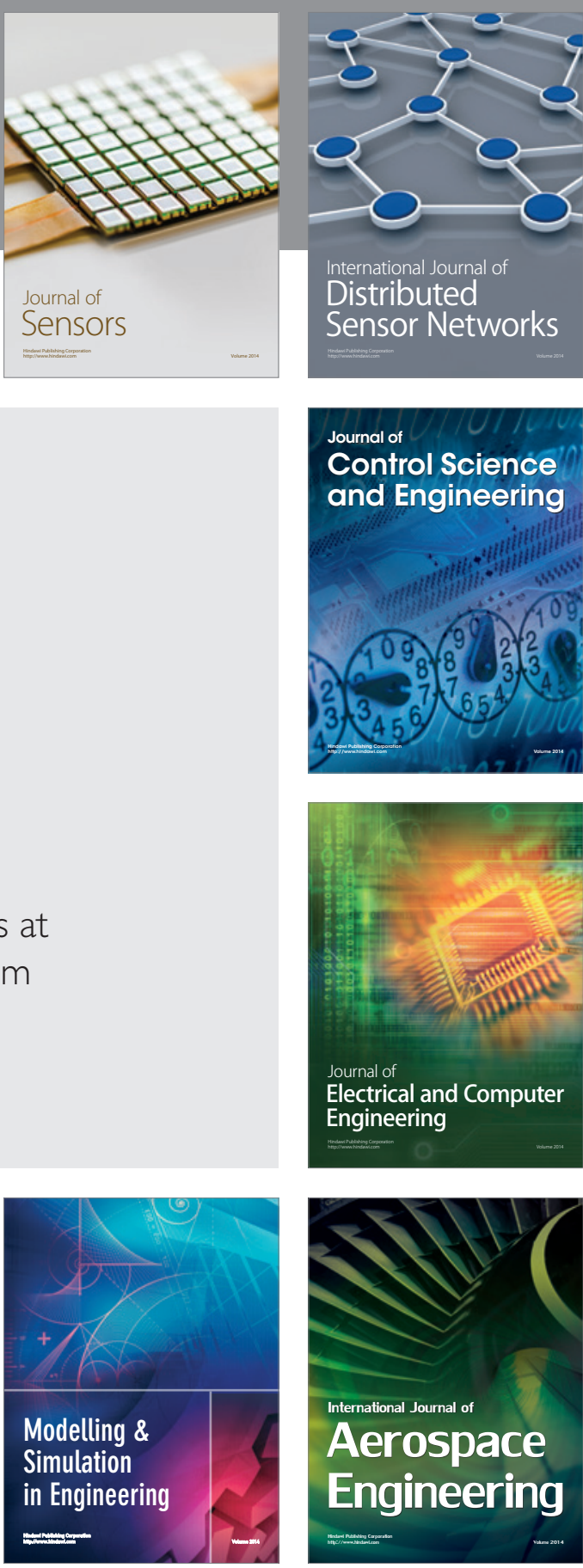

International Journal of

Distributed

Sensor Networks

$-$

Joumal of

Control Science

and Engineering
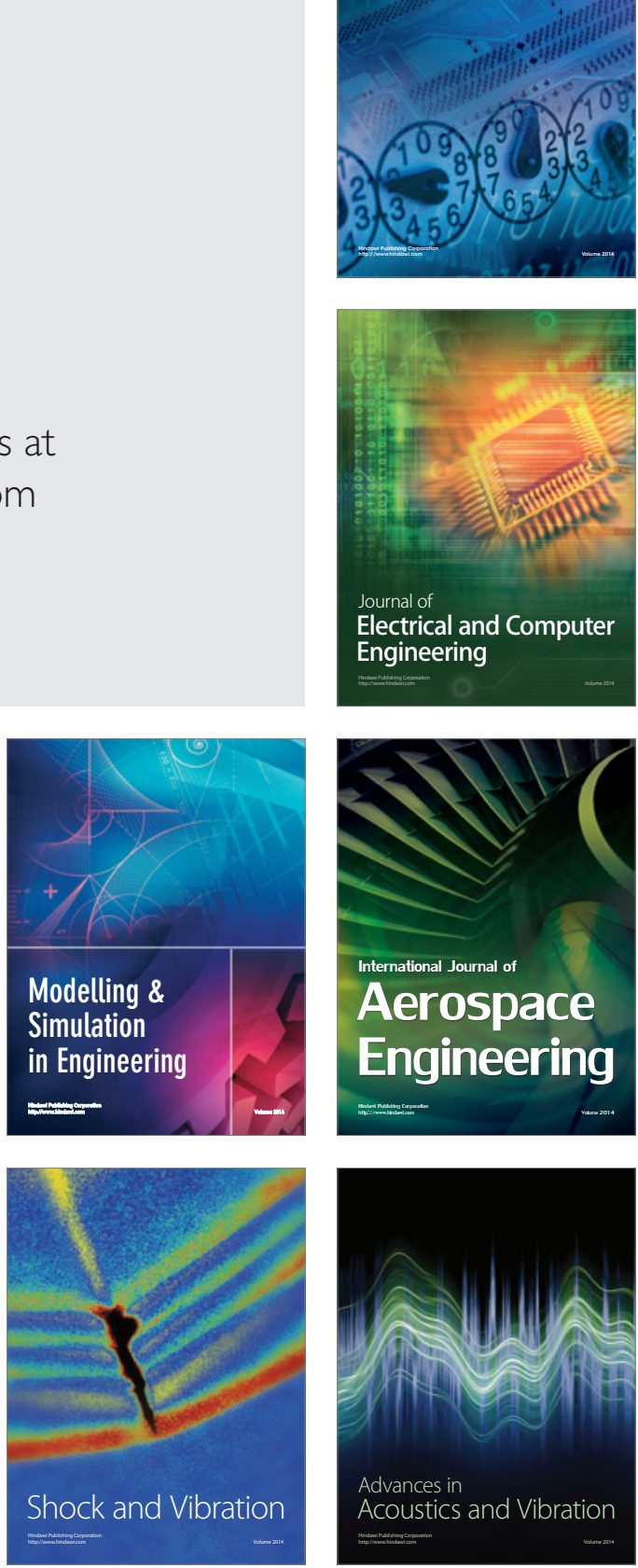